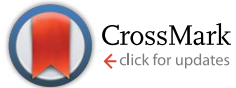

Cite this: RSC Adv., 2017, 7, 14966

Received 26th December 2016 Accepted 28th February 2017

DOI: 10.1039/c6ra28715h

rsc.li/rsc-advances

\section{Emerging role and recent applications of metabolomics biomarkers in obesity disease research}

\begin{abstract}
Aihua Zhang, ${ }^{a}$ Hui Sun ${ }^{a}$ and Xijun Wang ${ }^{\star a b}$
Metabolomics is a promising approach for the identification of metabolites which serve for early diagnosis, prediction of therapeutic response and prognosis of disease. Obesity is becoming a major health concern in the world. The mechanisms underlying obesity have not been well characterized; obviously, more sensitive biomarkers for obesity diseases are urgently needed. Fortunately, metabolomics - the systematic study of metabolites, which are small molecules generated by the process of metabolism - has been important in elucidating the pathways underlying obesity-associated co-morbidities. The endogenous metabolites have key roles in biological systems and represent attractive candidates to understand obesity phenotypes. Novel metabolite markers are crucial to develop diagnostics for obesity related metabolic disorders. Metabolomics allows unparalleled opportunities to seize the molecular mechanisms of obesity and obesity-related diseases. In this review, we discuss the current state of metabolomic analysis of obesity diseases, and also highlight the potential role of key metabolites in assessing obesity disease and explore the interrelated pathways.
\end{abstract}

\section{Introduction}

Approximately 1.5 billion people worldwide are overweight or affected by obesity, and the prevalence of obesity has dramatically increased and poses a major threat to human health. ${ }^{1}$ The World Health Organization has reported an average increase in the prevalence of overweight and obesity of approximately $0.3-$ $0.8 \%$ per year. ${ }^{2}$ The prevalence of obesity and its associated comorbidities including type 2 diabetes mellitus, dyslipidemia, cardiovascular disease, hypertension, and some cancers, has steadily increased over the last 40-50 years. ${ }^{4,5}$ More than 700 million will be obese worldwide by $2015 .^{3}$ Given that the prevalence of obesity has been increasing at an alarming rate in the last few decades, it is of crucial importance to understand the mechanisms underlying this process. It ultimately would help not only to reduce the economic burden of obesity but also to improve health outcomes and quality of life. Importantly, vast efforts of current research are needed towards understanding the origins of obesity and its underlying mechanisms.

Emerging metabolomics can be considered most closely related to a patient's phenotype. ${ }^{6}$ As this field advances, small-

\footnotetext{
${ }^{a}$ Metabolomics Laboratory, Sino-America Chinmedomics Technology Collaboration Center, National TCM Key Laboratory of Serum Pharmacochemistry, Chinmedomics Research Center of State Administration of TCM, Department of Pharmaceutical Analysis, Heilongjiang University of Chinese Medicine, Heping Road 24, Harbin 150040, China. E-mail: xijunwangls@126.com; Fax: +86-451-82110818; Tel: +86451-82110818

${ }^{b}$ State Key Laboratory of Quality Research in Chinese Medicine, Macau University of Science and Technology, Avenida Wai Long, Taipa, Macau, China
}

molecule metabolites have an important role in biological systems and represent attractive candidates to understand obesity phenotypes. They are crucial components of biological systems and highly informative about their functional state, due to their closeness to functional endpoints and to the organism's phenotypes. ${ }^{7}$ Metabolomics is useful for understanding the biochemical changes in obesity or related diseases. ${ }^{8}$ Metabolomics in identifying biomarkers or defining pathological status, has been growing exponentially as evidenced by the number of published papers. ${ }^{9}$ This article provides a brief overview of recent advances in metabolomics as applied to biomarker discovery and the elucidation of mechanisms underlying obesity and its co-morbidities, with specific emphasis on metabolite markers for the prevention and control of obesity.

\section{Basic methods and techniques of metabolomics}

A scheme of the metabolomics workflow is shown in Fig. 1. Nuclear Magnetic Resonance (NMR) spectroscopy and highresolution mass spectrometry (MS) are the main analytical platforms for metabolomics. ${ }^{\mathbf{1 0}}$ NMR has many advantages, but the sensitivity of NMR is relatively poor compared with MS methods, and concentrations of metabolite species may be below the detection limit. Each technique has associated advantages and disadvantages, and no single analytical methodology is ideal for all the metabolites in biological samples. A 


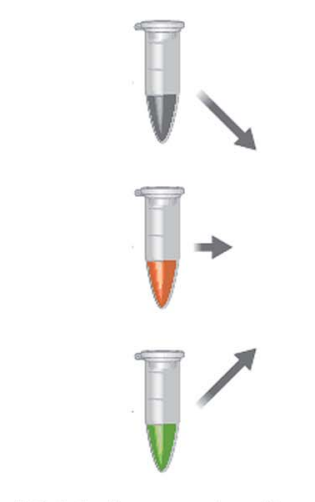

Metabolome extraction
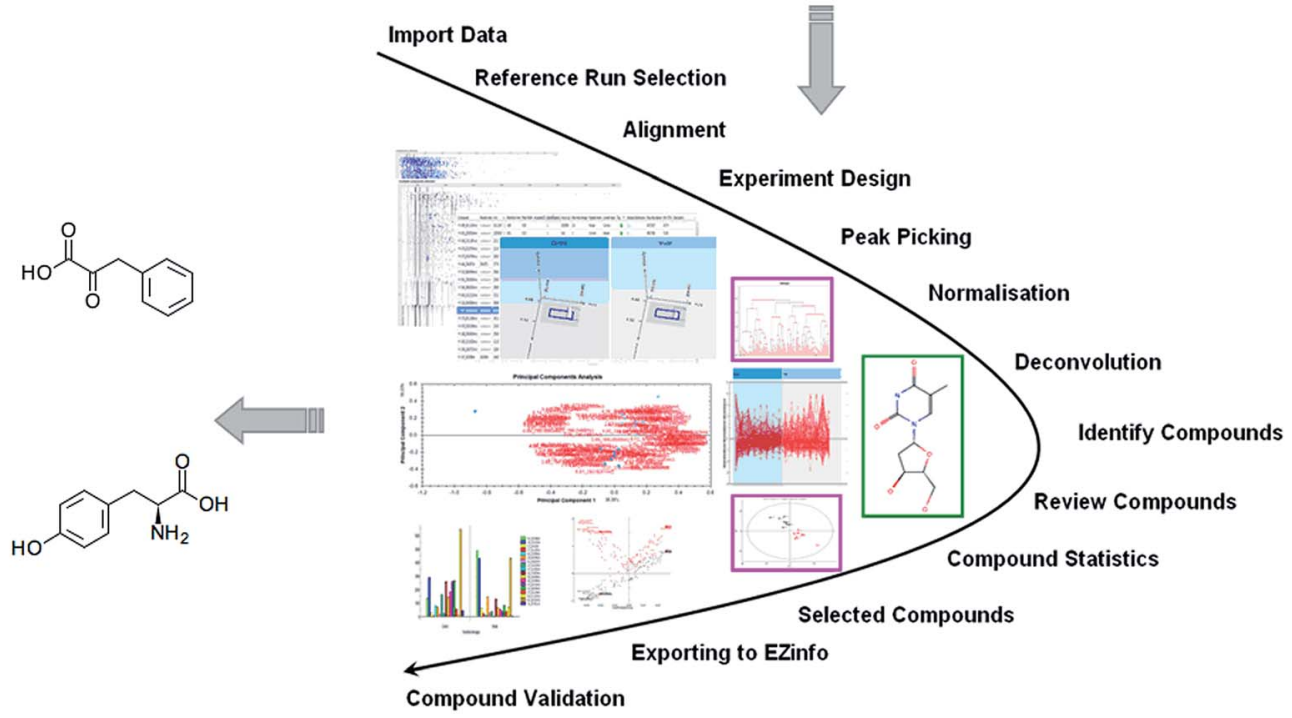

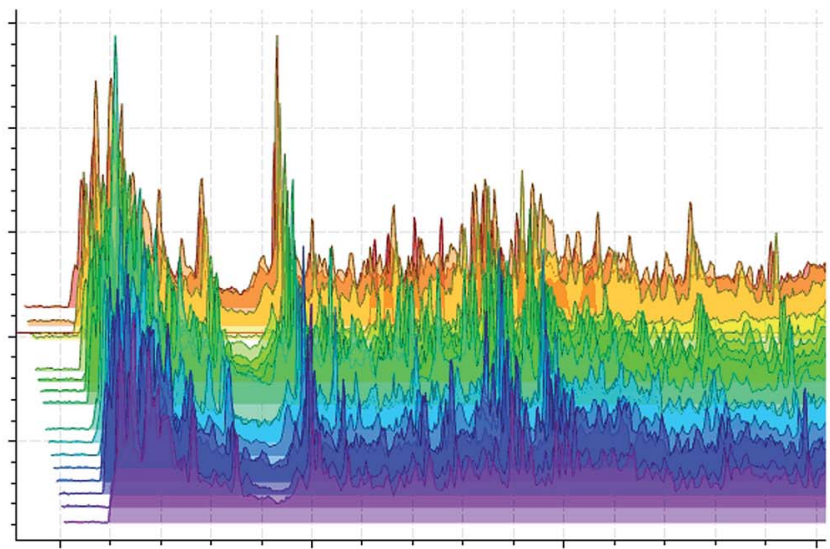

Total ion chromatogram analysis

Targeted or untargeted analysis of the data

Fig. 1 A scheme of the metabolomics workflow.

combination of advanced and high-throughput separation and analytical technologies could gain a broad perspective of the metabolome. Integrated NMR and MS with pattern recognition methods have driven forward the application of metabolomics in the field of metabolites discovery. Metabolic profiling of biosamples could provide a view of abundance changes of endogenous metabolites in responses to disease perturbations. Using pattern recognition approaches such as principal component analysis (PCA) and "supervised" partial least squares-discriminant analysis (PLS-DA) could provide a comprehensive assessment of the metabolites that are associated with the state of diseases. PCA score plot indicates the scatter of the samples, which indicate similar metabolomic compositions when clustered together and compositionally different metabolome when dispersed. PLS-DA attempts to maximize the covariance between the classes better than PCA. Potential biomarkers were extracted from loading-plot or variable importance in projection (VIP)-plot following PLS-DA, based on their contribution to the variation within the data set.

\section{Potential role of metabolomics}

At the end of the 20th century, genomics wrote out the 'script of life'; proteomics decoded the script; and metabolomics came into bloom. These 'omics' quickly became the thrust of life sciences, pushing the discipline to new high. Metabolomics is coming of age as an important area of investigation which may help reveal answers to questions left unanswered from proteomic or genomic approaches. The most advantages of metabolomics are the size of the metabolome relative to the genome or proteome and the fact that it provides a view of the existing biochemical phenotype. ${ }^{11}$ As such, metabolomics is fast becoming an important discovery tool for new diagnostic and prognostic biomarkers. In order to avoid false expectation by 
using a metabolomics approach, it is important to have: (1) a properly planned design of the study, (2) a proper statistical analysis and support, (3) a validation of the results. Deciphering information on metabolites in the complex biological systems requires not only sophisticated LC-MS systems, but also software that can help identify and quantify metabolites in the data. Fortunately, high-resolution MS technology has augmented the development of integrated data acquisition methods that includes various untargeted and targeted multivariate data analysis methods, which allowed the analysis of a wide range of biomolecules and significantly facilitated metabolite detection and identification. ${ }^{\mathbf{1 2}}$ Currently, metabolomics has been employed in translational research to help identify biologically relevant pathways and pathophysiology. ${ }^{13}$ The molecular pathways in diseases will lead to the discovery of new candidate biomarkers in clinical practice.

\section{Understanding action mechanisms by metabolomics}

Metabolomics is the study of metabolic changes in biological systems and provides the small molecule fingerprints related to the disease. For examples, metabolomics has a great and largely untapped potential in the field of oncology and the analysis of the cancer metabolome to identify biofluid markers and druggable targets. ${ }^{14}$ Several studies have applied metabolomic approach to identify the metabolite signatures. However, before translating a potential metabolite biomarker into clinical use, additional validation studies are required. Validation of biomarkers and targets may entail intensive use of labor and technology and generally requires a large number of study participants. Technological developments have enabled the identification and quantitative measurement of the many metabolites in a nontargeted and non-destructive manner. Metabolomics is fast, noninvasive, and sensitive tool for determining specific pathway utilization and provides a direct translational application in the health care field. ${ }^{15}$ Combining metabolomics with multivariate data analysis allows us to study alterations in metabolic pathways following different perturbations. Recent developments have suggested that metabolite profiles will confer a high degree of predictive accuracy in terms of understanding the fundamental mechanisms resulting in perturbations of the metabolic state. ${ }^{\mathbf{1 6}}$ Metabolomics involves the establishment of relationships between phenotype and metabolic signatures, which are key aspects of biological function. ${ }^{17}$ Scientific information derived from the metabolomics techniques can be applied to provide accurate and clinically useful diagnostic capability for the management of obesity and obesity-related diseases.

\section{Metabolomics as a diagnostic tool in obesity}

Recently, WHO indicate that the prevalence of obesity is not just a problem of the developed countries but is also on the increase in the world. The prevalence of obesity is rising alarmingly among children and adolescents, and obesity has become a major public health problem worldwide. ${ }^{18}$ Obesity is significant growing health problems in children, and is threatening their physical and mental health. In depth understanding of the mechanism of obesity could provide an opportunity to be most beneficial for addressing this problem. However, the mechanisms underlying the association between obesity and comorbidities are not well understood. There is still a lack of reliable biomarkers indicative of metabolic alterations associated with obesity and complications, highlighting the dire need for the development of early diagnostic and prognostic markers. Metabolomics has refreshed interest in metabolism across biology and medicine, particularly in the areas of functional genomics and biomarker discovery. ${ }^{19}$ It offers potential advantages in sensitivity and specificity, compared to classical diagnostic approaches and conventional clinical biomarkers. Studies with improved technology are needed to identify better biomarkers and targets for treatment or prevention of obesity. The process of metabolomics for obesity was shown in Fig. 2. Future application of metabolomics will add new perspectives to better understand the complexities of obesity.

\section{Metabolomics application in obesity}

Recent developments have suggested that metabolomics will play an increasing role in understanding the complexities of obesity, and will confer a high degree of understanding the fundamental mechanisms in perturbations of obesity states. Obesity can cause hyperlipidemia, cardiovascular diseases, metabolic syndrome and non-alcoholic fatty liver disease. A study showed a nontargeted metabolomic approach can be used to reveal the metabolites of obesity-related metabolic disturbances. ${ }^{20}$ Another study had identified key metabolites related to obesity in humans by studying the metabolic profiles of sera. ${ }^{21}$ The levels of betaine, pyroglutamic acid, pipecolic acid, $N$-phenylacetamide, uric acid etc., showed significantly increased. Particularly, lysophosphatidylcholine 16:0 with a higher VIP value is esteemed to be the most important metabolite. A recent study by Wang et al. was to examine changes in the plasma metabolites in obese adults. ${ }^{22}$ Obese and normal-weight groups were clearly discriminated on score plot and nine major metabolites contributing to the discrimination were assigned, such as increased 2-octenoylcarnitine, eicosadienoic acid, thromboxane B2 and pyridinoline and decreased vitamin D3 glucosiduronate, etc. One study reported that major metabolic processes like fatty acid biosynthesis, phenylalanine metabolism, propanoate metabolism, and valine, leucine and isoleucine degradation were altered in abnormal obesity. ${ }^{23} \mathrm{~A}$ study focused on the associations between metabolite profiles and phenotype parameters in plasma/serum samples from healthy overweight men and women with central obesity. ${ }^{24}$ The evidence indicates the potential of metabolomics in obesity and gender differences need to be taken into account for biomarker and diagnostics.

Targeted serum metabolomics identified 12 metabolites significantly related to obesity. ${ }^{25}$ The purpose of a recent study 
by Kim et al. was to examine the differences in metabolomic profiling between overweight/obese and normal-weight men. ${ }^{26}$ Overweight/obese men showed higher levels of triglycerides, total cholesterol, and LDL-cholesterol, and lower levels of HDLcholesterol and adiponectin than lean men. Especially, they identified three lyso-phosphatidylcholines as potential plasma markers and confirmed eight metabolites for overweight/ obesity men. Results confirmed abnormal metabolism of two branched-chain amino acids, two aromatic amino acids, and fatty acid synthesis and oxidation in overweight/obese men. With the use of an NMR-based metabolomic technology, serum lipids, unsaturated lipids, glycoprotein, myo-inositol, pyruvate, threonine, tyrosine and creatine were higher in obese, while serum glucose and urea were lower in obese. ${ }^{27}$ It indicated that obese has distinct metabolism, including lipogenesis, lipid oxidation, energy utilization and partition, protein and amino acid metabolism, etc.
Childhood obesity has become a prevalent risk to health of children and teenagers. Investigation of serum metabolites might give new insights into biological mechanisms associated with childhood obesity. Metabolite profiles of obese children could be distinguished from those of normal-weight children. ${ }^{28}$ After correction for multiple testing, Wahl et al., had observed 14 metabolites ratios to be significantly altered in obese children. The identified metabolite markers are indicative of oxidative stress and of changes in sphingomyelin metabolism and pathways associated with energy expenditure. There is sufficient evidence to conclude that childhood obesity influences the composition of the serum metabolome. A study investigated the differences of metabolomic profiling between normal-weight young men and obese with hyperlipidemia which is strongly related to the occurrence and development of obesity-related diseases. ${ }^{29}$ Eight urine principal metabolites contributing to the clusters were identified; they included increased L-prolyl-L-proline, leucyl-phenylalanine, decanoylcarnitine, $N$-acetylornithine, 11 $\beta$-hydroxyprogesterone,
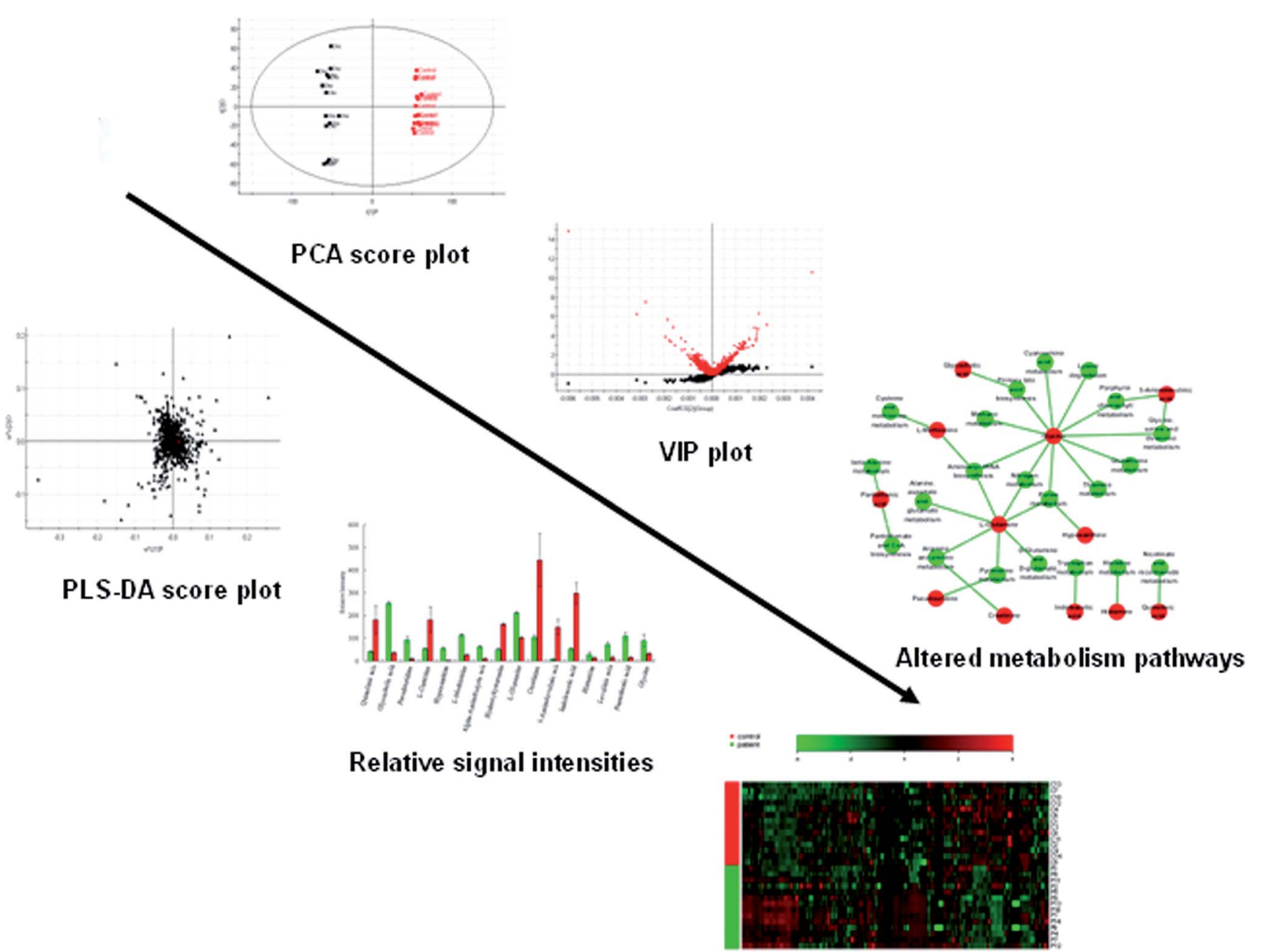

\section{Clinical Estimation of Metabolic Biomarkers}

Fig. 2 The process of metabolomics applications for investigating the pathophysiological mechanisms of obesity diseases. Samples collected by uniform way to minimise variability, are analysed by full scan MS profile or NMR to collect data on all potentially metabolites. Pattern recognition approach includes PCA, PLS-DA, VIP plot and other mode aiming to highlight underlying trends. Heatmap is generally considered the method of choice for evaluating the performance of potential biomarkers. The markers are eventually placed in altered metabolism pathways to provide insight on the biochemical phenomena. 


\section{A}

A 200

$\bar{T}$

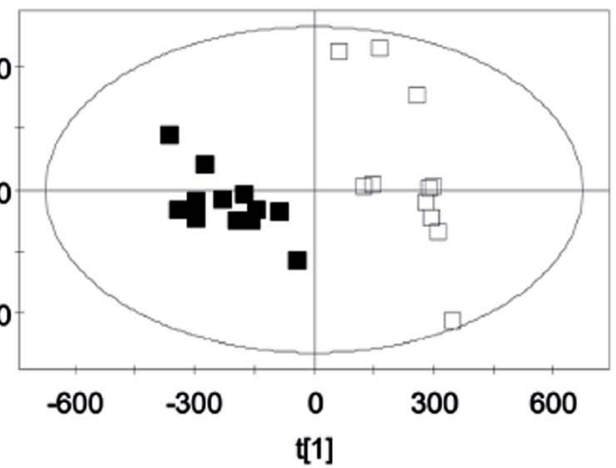

Lean mice
B

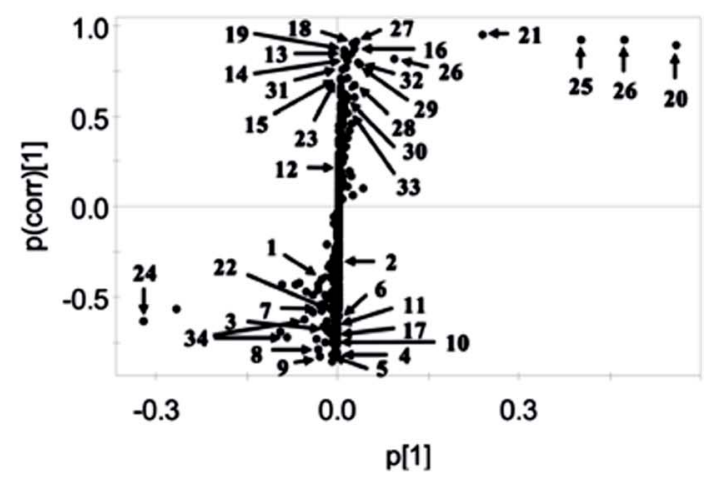

C

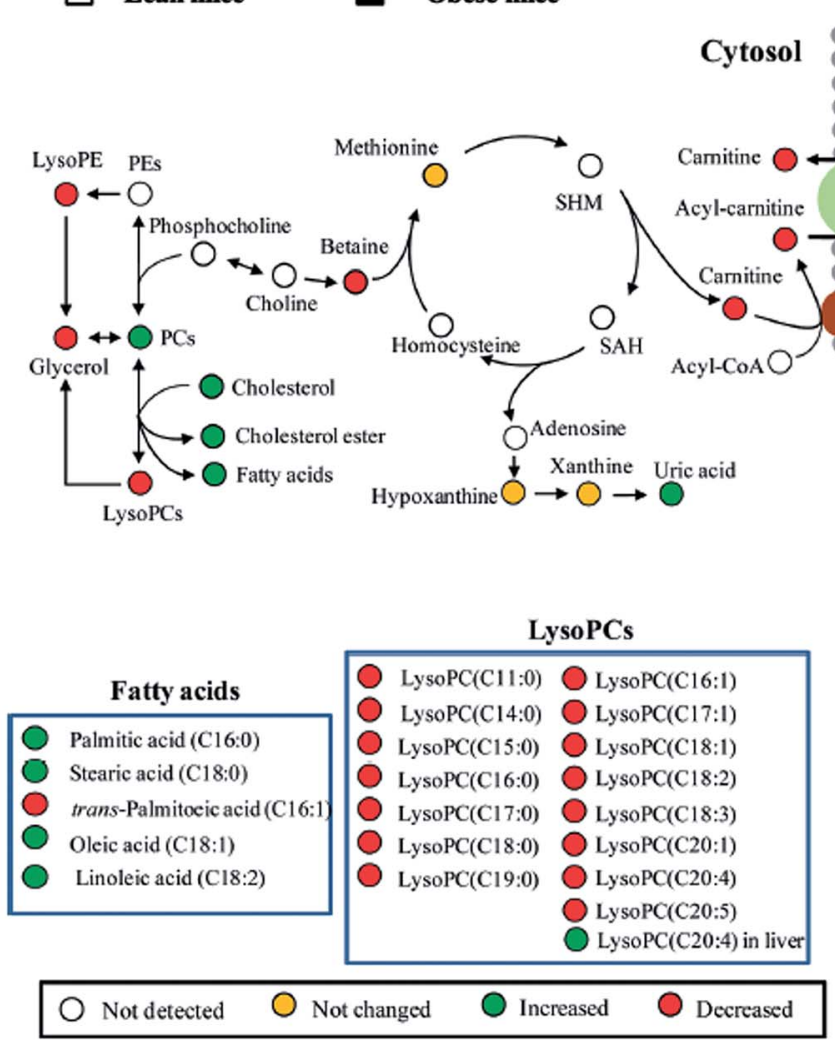

드를 Mitochondria

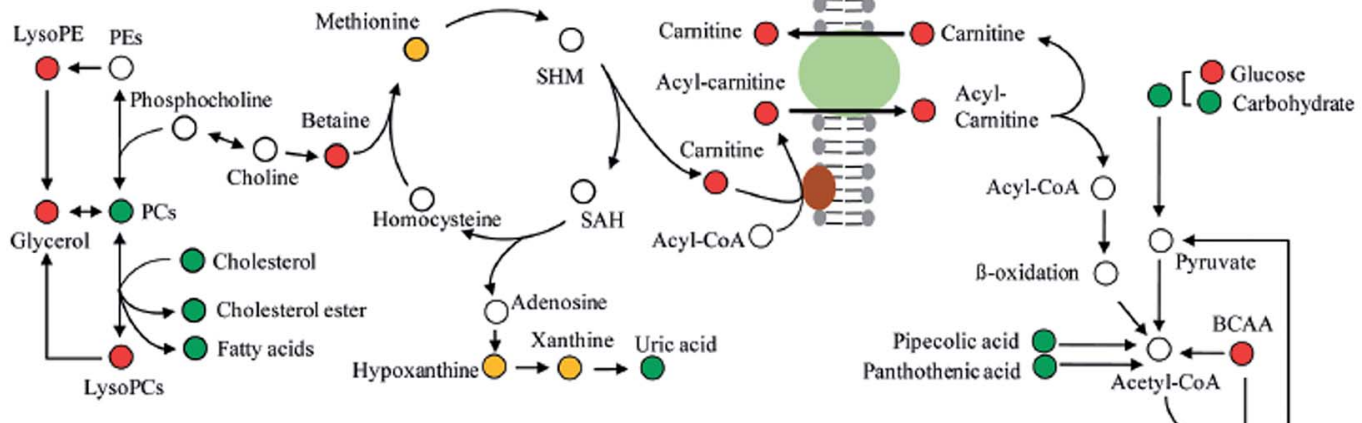

LysoPCs

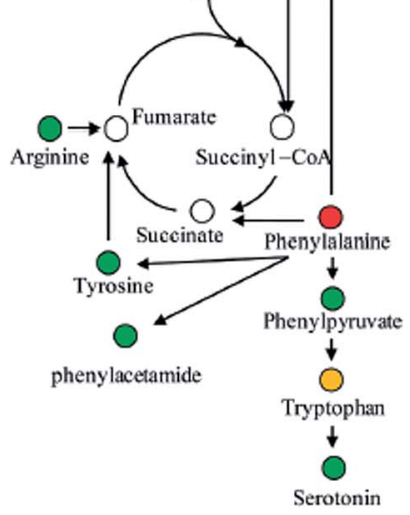

Fig. 3 Metabolomic analysis of high-fat diet induced obese mice. (A) Partial least-squares-discriminant analysis (PLS-DA) score plots obtained from UPLC-Q-TOF MS data of serum; (B) S-plots associated with the PLS-DA score plots of metabolite profiles derived from serum; (C) schematic diagram of the metabolic pathway to obesity induced by a high-fat diet based on serum metabolites analysis. Red represents increased metabolites, green represents decreased metabolites, yellow represents no change, and the open circles represent no detected metabolites.

$5 a$-dihydrotestosterone sulfate and glucosylgalactosyl hydroxylysine. These metabolite changes suggested early changes of metabolism in young-male obesity with hyperlipidemia, may further aid the clinical prevention and treatment of obesity and related diseases.

In another study, Duggan et al. profiled distinct metabolites of obesity by targeted metabolomics. ${ }^{30}$ Differences in metabolite levels were determined. Evidence showed that the importance of dietary controls and highlighted the ability of metabolomics to identify subtle changes. Obese and lean groups were clearly discriminated on PLS-DA score plot and major metabolites contributing to the discrimination were assigned as lipid metabolites, and lysophosphatidylcholines, lipid metabolism intermediates, amino acids, acidic compounds, monosaccharides, and serotonin. ${ }^{31}$ In addition, the key findings were that the levels of many metabolites were positively or negatively related to obesity-associated diseases. The level changes of these metabolites may be used to better understand obesity, assess the risk of obesity and therapeutic effect of obesity. The schematic diagram of the metabolic pathway to obesity induced by a high-fat diet based on serum metabolites analysis was shown in Fig. 3.

Martin et al. had examined the relationships between different body composition parameters, estimates of visceral 
adiposity and blood/urine metabolic profiles. ${ }^{32}$ A recent study compares the patterns of visceral (VIS) and subcutaneous (SC) adipose tissue (AT)-derived metabolites from non-obese and obese subjects. ${ }^{33}$ Differences related to obesity and AT were assessed using an untargeted metabolomics approach. Higher released levels of glutamine and alanine were detected in the VIS-obese AT, whereas the VIS-obese AT presented a diminished uptake of essential amino acids, branched-chain amino acids (BCAAs), leucine, and serine. This study showed the obesity markedly affects the amino acid metabolic signature of the AT before the clinical onset of the significant metabolic alterations. BCAAs are circulating nutrient signals for protein accretion, however, they increase in obesity and elevations appear to be prognostic of diabetes. To understand the mechanisms whereby obesity affects BCAAs and protein metabolism, She et al. had employed metabolomics measured rates of [1-(14)C]leucine metabolism, tissue-specific protein synthesis and branched-chain keto-acid (BCKA), dehydrogenase complex activities. ${ }^{34}$ The authors showed that urinary concentrations of eight BCAA catabolism-derived acylcarnitines were elevated. Furthermore, the authors found that plasma BCKAs may represent a more sensitive metabolic signature for obesity than BCAAs. Similarly, a review by Morris C. et al., found that there is an alterations in the amino acid profiles are associated with obesity. ${ }^{35}$ Several other studies have also shown a relationship between BCAA, obesity and insulin resistance. ${ }^{36}$

Won et al. reported metabolomic analysis of gender bias in obesity. ${ }^{37}$ The distinct metabolites are involved in amino acid metabolism, tricarboxylic acid cycle and glucose metabolism, lipid metabolism, creatine metabolism, and gut-microbiomederived metabolism. The authors provide biochemical insights into the metabolic alteration in obesity based on gender. Zhu et al. established a urinary metabolomic method to evaluate metformin action in preventing obesity. ${ }^{38}$ Significantly decreased glucose, myristic acid, stearidonic acid, etc. in urine indicated that metformin may have exerted effects on energy metabolism. Further, published data showed that tricarboxylic acid cycle may have been compromised by metformin and might contribute to the activation of adenosine monophosphate kinase (AMPK), and then AMPK activation led to more $\beta$-oxidation of certain fatty acids and augmented lipolysis and thus induced weight loss. Ma B. et al. had explored and identify the alteration of endogenous metabolites using metabolomic approach in a rat model of estrogen-deficiencyinduced obesity. ${ }^{39}$ Elevated levels of cholesterol, glycerol, glucose, arachidonic acid, glutamic acid, glycine, and cystine and reduced alanine levels were observed, and involved glucose metabolism, lipid metabolism, and amino acid metabolism.

In another study, Sampey et al. used metabolomics to identify potential mediators of obesity and metabolic syndrome. ${ }^{40}$ PCA identified elevations in clusters of fatty acids and acylcarnitines. These increases in metabolites were associated with systemic mitochondrial dysfunction. Further analysis result showed that modifying the availability or metabolism of saturated fatty acids may limit the inflammation associated with obesity leading to metabolic syndrome. Published data showed that gut flora-derived metabolites such as hippuric acid, 2- hydroxyisobutyrate, trigonelline and xanthine were responsible for the obesity-associated metabolic phenotype. ${ }^{41}$ These data suggest that gut microflora metabolism in humans is strongly linked to the obesity phenotype. UHPLC-MS and multivariate analyses were utilized to analyze obesity related metabolites in rat urine samples ${ }^{42}$. It successfully identified 20 altered metabolites in urine that correlated with obesity. These metabolites were involved in phenylalanine and tyrosine metabolism, tryptophan metabolism, and gut microbiota metabolism related metabolism.

\section{Conclusions and future perspectives}

Obesity is currently epidemic in many countries worldwide and there is an urgent need to identify molecular markers that will enable early detection of the pathophysiological processes leading to the co-morbidities of obesity. Metabolomics is capable of describing the biochemical phenotype of a biological system, and also provides a basis for the prevention or treatment of obesity. Metabolite profile is reflective of metabolic processes, including pathophysiological changes characteristic of obesity-related complications. Furthermore, clarifying intermediate phenotypes and their markers might lead to novel therapeutic and diagnostic strategies for obesity-associated comorbidities. Herein, the authors review how metabolomics can produce novel mechanistic insights, identify potential biomarkers from metabolome datasets and uncover interpretation of the perturbed metabolic processes in the obesity and obesity-related complications. We have showed examples of how metabolomics provides an insight to the metabolite markers in obesity, always keeping in mind its potential application in obesity-related complications areas as a biomarker discovery tool. If replicated in larger studies at a population level, the altered metabolites might be considered as potential biomarkers in the generation of new hypotheses on the biological mechanisms behind obesity.

\section{Competing financial interests}

The authors declare no competing financial interests.

\section{Acknowledgements}

This work was supported by grants from the Key Program of Natural Science Foundation of State (Grant no. 81430093, 81302905, 81373930, 81673586), National Key Subject of Drug Innovation (Grant no. 2015ZX09101043-005, 2015ZX09101043011), TCM State Administration Subject of Public Welfare of (Grant no. 2015468004), University Nursing Program for Young Scholars with Creative Talents in Heilongjiang Province (UNPYSCT-2015118).

\section{References}

1 S. Wahl, A. Drong, B. Lehne, M. Loh, W. R. Scott, S. Kunze, et al. Epigenome-wide association study of body mass 
index, and the adverse outcomes of adiposity, Nature, 2017, 541(7635), 81-86.

2 E. Kupek, A. S. Lobo, D. B. Leal, F. Bellisle and M. A. de Assis, Dietary patterns associated with overweight and obesity among Brazilian schoolchildren: an approach based on the time-of-day of eating events, Br. J. Nutr., 2016, 116(11), 1954-1965.

3 M. Okada-Iwabu, T. Yamauchi, M. Iwabu, et al. A smallmolecule AdipoR agonist for type 2 diabetes and short life in obesity, Nature, 2013, 503(7477), 493-499.

4 S. Yoshimoto, T. M. Loo, K. Atarashi, et al. Obesity-induced gut microbial metabolite promotes liver cancer through senescence secretome, Nature, 2013, 499(7456), 97-101.

5 D. T. Phung, Z. Wang, S. Rutherford, C. Huang and C. Chu, Body mass index and risk of pneumonia: a systematic review and meta-analysis, Obes. Rev., 2013, 14(10), 839-857.

6 J. C. Schoeman, J. Hou, A. C. Harms, R. J. Vreeken, R. Berger, T. Hankemeier and A. Boonstra, Metabolic characterization of the natural progression of chronic hepatitis $\mathrm{B}$, Genome Med., 2016, 8(1), 64.

7 S. G. Musharraf, A. J. Siddiqui, T. Shamsi and A. Naz, SERUM metabolomics of acute lymphoblastic leukaemia and acute myeloid leukaemia for probing biomarker molecules, Hematol. Oncol., 2016, DOI: 10.1002/hon.2313.

8 Y. Zhang, E. Guallar, E. Blasco-Colmenares, A. C. Harms, R. J. Vreeken, T. Hankemeier, G. F. Tomaselli and A. Cheng, Serum-Based Oxylipins are Associated with Outcomes in Primary Prevention Implantable Cardioverter Defibrillator Patients, PLoS One, 2016, 11(6), e0157035.

9 A. Zhang, H. Sun and X. Wang, Power of metabolomics in biomarker discovery and mining mechanisms of obesity, Obes. Rev., 2013, 14(4), 344-349.

10 A. H. Zhang, H. Sun, S. Qiu and X. J. Wang, NMR-based metabolomics coupled with pattern recognition methods in biomarker discovery and disease diagnosis, Magn. Reson. Chem., 2013, 51(9), 549-556.

11 A. Zhang, H. Sun and X. Wang, Mass spectrometry-driven drug discovery for development of herbal medicine, Mass Spectrom. Rev., 2016, DOI: 10.1002/mas.21529.

12 X. Wang, A. Zhang, P. Wang, et al. Metabolomics coupled with proteomics advancing drug discovery toward more agile development of targeted combination therapies, Mol. Cell. Proteomics, 2013, 12(5), 1226-1238.

13 A. Zhang, H. Sun and X. Wang, Mass spectrometry-driven drug discovery for development of herbal medicine, Mass Spectrom. Rev., 2016, DOI: 10.1002/mas.21529.

14 H. Sun, A. Zhang, G. Yan, et al. Metabolomic analysis of key regulatory metabolites in hepatitis $\mathrm{C}$ virus-infected tree shrews, Mol. Cell. Proteomics, 2013, 12(3), 710-719.

15 X. Wang, A. Zhang and H. Sun, Power of metabolomics in diagnosis and biomarker discovery of hepatocellular carcinoma, Hepatology, 2013, 57(5), 2072-2077.

16 D. Q. Tang, L. Zou, X. X. Yin and C. N. Ong, HILIC-MS for metabolomics: an attractive and complementary approach to RPLC-MS, Mass Spectrom. Rev., 2016, 35(5), 574-600.

$17 \mathrm{X}$. Wang, A. Zhang, Y. Han, et al. Urine metabolomics analysis for biomarker discovery and detection of jaundice syndrome in patients with liver disease, Mol. Cell. Proteomics, 2012, 11(8), 370-380.

18 M. M. Khamis, D. J. Adamko and A. El-Aneed, Mass spectrometric based approaches in urine metabolomics and biomarker discovery, Mass Spectrom. Rev., 2017, 36(2), 115-134.

19 A. Zhang, H. Sun, P. Wang, Y. Han and X. Wang, Modern analytical techniques in metabolomics analysis, Analyst, 2012, 137(2), 293-300.

20 K. Hanhineva, T. Barri, M. Kolehmainen, et al. Comparative nontargeted profiling of metabolic changes in tissues and biofluids in high-fat diet-fed Ossabaw pig, J. Proteome Res., 2013, 12(9), 3980-3992.

21 M. J. Kim, H. J. Yang, J. H. Kim, et al. Obesity-related metabolomic analysis of human subjects in black soybean peptide intervention study by ultra performance liquid chromatography and quadrupole-time-of-flight mass spectrometry, J. Obes., 2013, 2013, 874981.

22 Y. Wang, D. Liu, Y. Li, L. Guo, Y. Cui, X. Zhang and E. Li, Metabolomic analysis of serum from obese adults with hyperlipemia by UHPLC-Q-TOF MS/MS, Biomed. Chromatogr., 2016, 30(1), 48-54.

23 H. H. Chen, Y. J. Tseng, S. Y. Wang, Y. S. Tsai, C. S. Chang, T. C. Kuo, W. J. Yao, C. C. Shieh, C. H. Wu and P. H. Kuo, The metabolome profiling and pathway analysis in metabolic healthy and abnormal obesity, Int. J. Obes., 2015, 39(8), 1241-1248.

24 E. Szymańska, J. Bouwman, K. Strassburg, et al. Genderdependent associations of metabolite profiles and body fat distribution in a healthy population with central obesity: towards metabolomics diagnostics, OMICS, 2012, 16(12), 652-667.

25 A. Oberbach, M. Blüher, H. Wirth, et al. Combined proteomic and metabolomic profiling of serum reveals association of the complement system with obesity and identifies novel markers of body fat mass changes, $J$. Proteome Res., 2011, 10(10), 4769-4788.

26 J. Y. Kim, J. Y. Park, O. Y. Kim, et al. Metabolic profiling of plasma in overweight/obese and lean men using ultra performance liquid chromatography and Q-TOF mass spectrometry (UPLC-Q-TOF MS), J. Proteome Res., 2010, 9(9), 4368-4375.

27 Q. He, P. Ren, X. Kong, et al. Comparison of serum metabolite compositions between obese and lean growing pigs using an NMR-based metabonomic approach, J. Nutr. Biochem., 2012, 23(2), 133-139.

28 S. Wahl, Z. Yu, M. Kleber, et al. Childhood obesity is associated with changes in the serum metabolite profile, Obes. Facts, 2012, 5(5), 660-670.

29 C. Wang, R. Feng, D. Sun, Y. Li, X. Bi and C. Sun, Metabolic profiling of urine in young obese men using ultra performance liquid chromatography and Q-TOF mass spectrometry (UPLC/Q-TOF MS), J. Chromatogr. B: Anal. Technol. Biomed. Life Sci., 2011, 879(27), 2871-2876.

30 G. E. Duggan, D. S. Hittel, C. C. Hughey, et al. Differentiating short- and long-term effects of diet in the obese mouse using 
${ }^{1} \mathrm{H}$-nuclear magnetic resonance metabolomics, Diabetes, Obes. Metab., 2011, 13(9), 859-862.

31 H. J. Kim, J. H. Kim, S. Noh, et al. Metabolomic analysis of livers and serum from high-fat diet induced obese mice, $J$. Proteome Res., 2011, 10(2), 722-731.

32 F. P. Martin, I. Montoliu, S. Collino, et al. Topographical body fat distribution links to amino acid and lipid metabolism in healthy non-obese women, PLoS One, 2013, 8(9), e73445.

33 F. A. Hanzu, M. Vinaixa, A. Papageorgiou, et al. Obesity rather than regional fat depots marks the metabolomic pattern of adipose tissue: an untargeted metabolomic approach, Obesity, 2014, 22(3), 698-704.

34 P. She, K. C. Olson, Y. Kadota, et al. Leucine and protein metabolism in obese Zucker rats, PLoS One, 2013, 8(3), e59443.

35 C. Morris, C. O'Grada, M. Ryan, et al. The relationship between BMI and metabolomic profiles: a focus on amino acids, Proc. Nutr. Soc., 2012, 71(4), 634-638.

36 B. C. Batch, S. H. Shah, C. B. Newgard, et al. Branched chain amino acids are novel biomarkers for discrimination of metabolic wellness, Metabolism, 2013, 62(7), 961-969.

37 E. Y. Won, M. K. Yoon, S. W. Kim, et al. Gender-Specific Metabolomic Profiling of Obesity in Leptin-Deficient ob/ob
Mice by ${ }^{1} \mathrm{H}$ NMR Spectroscopy, PLoS One, 2013, 8(10), e75998.

38 Y. Zhu, Y. Feng, L. Shen, et al. Effect of metformin on the urinary metabolites of diet-induced-obese mice studied by ultra performance liquid chromatography coupled to timeof-flight mass spectrometry (UPLC-TOF/MS), J. Chromatogr. B: Anal. Technol. Biomed. Life Sci., 2013, 925, 110-116.

39 B. Ma, Q. Zhang, G. J. Wang, et al. GC-TOF/MS-based metabolomic profiling of estrogen deficiency-induced obesity in ovariectomized rats, Acta Pharmacol. Sin., 2011, 32(2), 270-278.

40 B. P. Sampey, A. J. Freemerman, J. Zhang, et al. Metabolomic profiling reveals mitochondrial-derived lipid biomarkers that drive obesity-associated inflammation, PLoS One, 2012, 7(6), e38812.

41 R. Calvani, A. Miccheli, G. Capuani, et al. Gut microbiomederived metabolites characterize a peculiar obese urinary metabotype, Int. J. Obes., 2010, 34(6), 1095-1098.

42 L. Men, Z. Pi, Y. Zhou, M. Wei, Y. Liu, F. Song and Z. Liu, Urine metabolomics of high-fat diet induced obesity using UHPLC-Q-TOF-MS, J. Pharm. Biomed. Anal., 2017, 132, 258266. 